\title{
Vision-based active safety system for automatic stopping
}

\author{
Vicente Milanés \\ David F. Llorca Jorge Villagrá \\ Joshue Pérez \\ Ignacio Parra Carlos González \\ Miguel A. Sotelo
}

\begin{abstract}
A B S T R A C T
Intelligent systems designed to reduce highway fatalities have been widely applied in the automotive sector in the last decade. Of all users of transport systems, pedestrians are the most vulnerable in crashes as they are unprotected. This paper deals with an autonomous intelligent emergency system designed to avoid collisions with pedestrians. The system consists of a fuzzy controller based on the time-to-collision estimate - obtained via a vision-based system - and the wheel-locking probability - obtained via the vehicle's CAN bus - that generates a safe braking action. The system has been tested in a real car - a convertible Citroën C3 Pluriel - equipped with an automated electro-hydraulic braking system capable of working in parallel with the vehicle's original braking circuit. The system is used as a last resort in the case that an unexpected pedestrian is in the lane and all the warnings have failed to produce a response from the driver.
\end{abstract}

\section{Introduction}

Road safety continues to be one of the sticking points for European governments. Even though the number of road accidents involving personal injury fell by some $12 \%$ between 1991 and 2007 (Commission, 2009), the European Transport Commission is working to improve these results.

A report by the national highway traffic safety administration in the United States indicated that about $80-90 \%$ of traffic crashes are caused by driver errors (N.C. for Statistics, Analysis, 2006). Accordingly, active vehicle safety has become a major topic of research in intelligent transportation systems (Castro, Delgado, \& Medina, 2011; Chang, Tsai, \& Young, 2010; Milanés, Pérez, Godoy, \& Onieva, 2012). Moreover, automobile manufacturers are ever more concerned about vehicle safety, and are increasing their efforts to find reliable solutions to the problem. Several intelligent systems are beginning to be included in commercial vehicles, such as Antilock Braking Systems (ABS), Collision Warning, Lane Keeping, 4 Wheel Active Steering (4WAS), and Active Suspension systems. With respect to longitudinal dynamics, Adaptive Cruise Control (ACC), mainly used on highways, is capable of detecting the speed and distance of the vehicle ahead and modifying the speed accordingly. In urban environments, Volvo has developed an intelligent system capable of stopping the car in the case of a rear-end collision at speeds below $15 \mathrm{~km} / \mathrm{h}$ in traffic jam situations.

The ability to quickly reduce speed in a stable manner is one of the vital functions of a vehicle. A large proportion of situations threatening the safety of a vehicle occur when the driver tries to stop the vehicle abruptly without taking into account the car's performance in specific environmental conditions (slippery surface, braking and cornering, $\mu$ split). This is why an anti-lock system seems to be mandatory in any emergency braking control algorithm.

Since vehicles exhibit highly nonlinear dynamics that are hard to model precisely for this kind of manœuvre, and pedestrians are the most vulnerable in crashes as they are unprotected, intelligent systems need to be developed in order to protect them.

Indeed, pedestrian protection is a key problem in the context of the automotive industry and its applications. Onboard sensor systems are required to predict the vehicle host-to-pedestrian (H2P) distance as well as the time-to-collision (TTC) (Milanés et al., 2012). Range-based sensors such as radar and laser provide very accurate distance and time-to-crash measurements, and they have been previously used by many authors (Gidel, Checchin, Blanc, Chateau, \& Trassoudaine, 2010). However they are too expensive solutions for automotive industry compared with digital cameras. So, cameras are the most commonly used sensor for this purpose. The use of video sensors comes quite naturally for the problem of pedestrian detection since they provide texture information which enables the use of quite discriminative pattern recognition techniques. The human visual perception system is perhaps the best example of what performance might be possible with such sensors, if the appropriate algorithm were used. In addition, the use of image data as a source of information fits in with other 
intelligent vehicle applications such as lane detection, vehicle detection, and autonomous driving.

From the computer vision perspective, pedestrian detection is a difficult task. Large variations in pedestrian appearance (e.g., clothing, pose, etc.) and in environmental conditions (e.g., lighting, moving background, etc.) make this problem particularly challenging. The first stage in most systems consists of identifying generic obstacles as regions of interest (ROIs) using prior scene knowledge (camera calibration, ground plane constraint, etc.) and a computationally efficient method. Subsequently, a more computationally expensive pattern recognition step is applied. The lack of explicit models leads to the use of machine learning techniques, where an implicit representation is learned from features obtained from thousands of samples. The following surveys provide both a general and a detailed background on work on pedestrian detection for automotive applications (Gandhi \& Trivedi, 2007; Moeslund, Hilton, \& Krüger, 2006; Poppe, 2007), including some of recent publication (Enzweiler \& Gavrila, 2003; Gerónimo, López, Sappa, \& Graf, 2010). Most work on human motion is summarized in Moeslund et al. (2006) and Poppe (2007). The focus of Gandhi and Trivedi (2007) and Gerónimo et al. (2010) is on the application of pedestrian protection in the intelligent vehicle domain, covering both passive and active safety techniques. Finally, an overview of the current state of the art both methodologically and experimentally is presented in Enzweiler and Gavrila (2003), in which a novel benchmark set has been made publicly available.

An intelligent system for pedestrian protection can be divided into three steps: pedestrian detection, assessment of the risk of collision, and, if appropriate, emergency stopping. The first two steps have to rely on a fast and reliable exteroceptive sensor. A vision-based algorithm able to detect pedestrians was developed in the present work. It will provide not only the distance to the obstacle, but also the level of confidence of that datum.

In this communication, we describe a fuzzy controller for an intelligent emergency stopping system. It uses as inputs the wheel-locking probability - obtained through on-board vehicle sensors via a Controller Area Network (CAN) bus - in order to optimize the braking distance and the TTC - obtained via a visionbased pedestrian detection system - to assess the risk in each case of emergency. The output is the braking pressure exerted on the wheels through an autonomous electro-hydraulic braking system installed in a Citroën C3 vehicle.

In brief, the main contributions of the work are the following:

- A vision-based intelligent emergency stopping control algorithm to avoid hitting pedestrians has been implemented in a real vehicle.
- The proposed control system is conceived to allow any car to be driven manually, with automatic assistance if necessary. Furthermore, the system implemented can easily be adapted and installed on any production car.

- A stereo vision-based pedestrian detection algorithm provides reliable and fast estimates of distance to the target.

- The braking action takes into account not only an estimated TTC from the camera, but also the probability of wheel-lock in order to improve the braking distance.

The rest of the article is organized as follows. Section 2 presents the hardware implementation and control architecture of the experimental vehicle. The pedestrian detection system will use two input variables: the wheel locking probability - presented in Section 3 and the estimation of TTC provided by the vision-based pedestrian detection system - described in Section 4. The resulting controller is described in Section 5. Section 6 presents the experimental results, and some concluding remarks are given in Section 7.

\section{Experimental prototype vehicle}

The experimental trials used a commercial mass-produced car equipped with automatic driving capabilities (see Fig. 1). This section briefly describes those modifications and explains the control architecture.

\subsection{Hardware implementation}

The autonomous braking system consists of a 1-1 capacity brake fluid tank that includes a gear pump and coupling to a 350-W, 12V supply, DC motor. Pressure control is implemented through three valves. The first is a spool directional valve to allow or stop the flow of brake fluid; the second is an electro-proportional pilot to regulate the nominal pressure; and the third is a pressure limiter tube - fixed at 160 bars - to avoid any excessive pressure being exerted in the wheels (Milanés, González, Naranjo, Onieva, \& de Pedro, 2010).

The automatic braking system is controlled via a CAN I/O device that is connected to the on-board control unit (OCU) where the control program is executed (a C++ application). The device includes a relay output to activate the spool directional valve and an analog output from 0 to $10 \mathrm{~V}$ to manage the electro-proportional pilot. Since this valve is oversized for this vehicle, the maximum allowed voltage is $6 \mathrm{~V}-60 \%$ of the valve's opening. The system was installed under the trunk in the place reserved for the spare wheel (Fig. 1).

Two cameras are used to detect pedestrians (Fig. 1). They are connected to a laptop via two FireWire ports. The cameras are

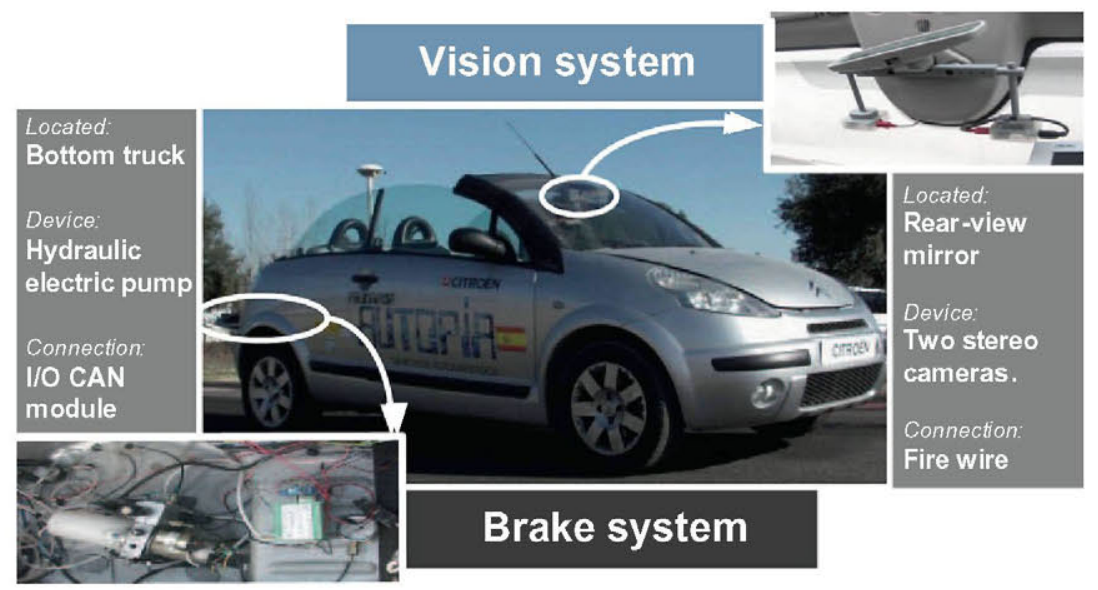

Fig. 1. Experimental prototype vehicle, on-board vision system, and automatic electro-hydraulic braking system. 
synchronized at $25 \mathrm{~Hz}$, with an image resolution of $320 \times 240$ pixels, a distance between the cameras (baseline) of $30 \mathrm{~cm}$ and a camera focal length of $8 \mathrm{~mm}$. The interconnection between the OCU and the laptop is via an Ethernet port to transmit the vision system output values.

\subsection{Control architecture}

The control architecture is based on a classical hierarchical system that is widely used in mobile robotics (Murphy, 2000). It naturally divides into three stages: perception, planning, and actuation (Fig. 2).

In the first stage - perception - all the sensorial inputs coming from the environment and the on-board vehicle sensors are acquired. The vehicle is equipped with a differential global positioning system (DGPS) and an inertial measurement unit (IMU) that are used for the vehicle's guidance (Milanés, Naranjo, González, Alonso, \& Pedro, 2008). The stereo vision system located in the rear-view mirror (Fig. 1) is responsible for pedestrian detection. The vehicle's CAN bus is designed to handle the acquisition of onboard sensor data such as wheel speed - coming from the four wheels - and the vehicle's acceleration.

In the second stage - planning - the control strategies are carried out. The decision system selects among different fuzzy controllers in order to perform the appropriate manœuvre as a function of the environmental data - provided by the first stage. The appropriate controllers to execute ACC (Onieva et al., 2010) or intersection manœuvres (Milanés, Pérez, Onieva, \& González, 2010) have been developed previously.

The last stage - actuation - executes the targets received from the planning stage, and modifies the outputs generated as voltage levels than can be sent to the actuators. Specifically, this is the value to apply to the electro-proportional pilot.

\section{Slip-based braking system}

During the acceleration or braking phases, the friction forces sgenerated $F_{x}$ are proportional to the normal load $F_{z}$ of the vehicle through the road adhesion coefficient $\mu$. Hence, knowledge of tyreroad forces is crucial, because optimal braking performance is related to the maximum tyre-road friction coefficient. Since determining these forces precisely is far from straightforward, one usually takes slip to be the decision variable in an anti-lock control system. It can be experimentally shown that $\mu$ is a nonlinear function of the wheel slip ratio $\tau$ (Liu, 2008; Zanten, Erhardt, \& Lutz, 1990) $\begin{cases}\tau=\frac{\omega r-V_{x}}{V_{x}} & \text { if } V_{x}>\omega r, V_{x}>0 \text { (braking) } \\ \tau=\frac{\omega r-V_{x}}{\omega r} & \text { if } V_{x}<\omega r, \omega r>0 \text { (accelerating) }\end{cases}$

As a result, many such control systems seek to ensure that the wheel slip ratio stays within a certain safety range, which is usually between 0.1 and 0.2 for most road conditions (cf. Zanten et al. (1990) for more details). In other studies, known optimal slip ratios for various road characteristics are used as reference signals (e.g., Wang, Chen, \& Tao, 2003; Wang, Li, Chen, Su, \& Hsu, 2009). However, the main problem with these methods is how to find the optimal slip ratio when the road characteristics are unknown or poorly known.

Although many attempts have been made, an accurate and general mathematical model of tyre behavior for any road conditions and driver action has yet to be obtained. Furthermore, at present there are no affordable sensors which can accurately identify the road surface and make this data available to an anti-lock controller (cf. Akbarzadeh, Emami, \& Pariz, 2004).

Many different control techniques have been applied to try to solve this complex problem, most of the times in a simulation environment. Examples are: model based adaptive control (Su, Chang, \& Chen, 2006) and sliding modes control (Kayacan, Oniz, \& Kaynak, 2009), fuzzy control (Mauer, 1995), neuro-fuzzy control (Wang et al., 2009), genetic neural control (Lee \& Zak, 2001). Fuzzy logic in particular seems to be an interesting choice because of its good compromise between tuning simplicity - contrarily to neuro-fuzzy and genetic neural control - and robustness to disturbances and parameter variations - it is independent of complex vehicle and brake models. It is well known (cf. Jamshidi, Vadiee, \& Ross, 1993) that the performance of fuzzy controllers can compare favorably with that of advanced model-based digital controllers. However, to the best of our knowledge, this intelligent control approach has yet to be implemented in real platforms (mass-production cars) for an emergency braking situation.

The results of experiments with our instrumented vehicle will be used to select the aforementioned slip safety ranges in order to provide an estimate of the probability of wheel lock.

\subsection{Experimental slip ratio ranges}

Several tests were performed with the experimental platform described in Section 2. The vehicle accelerates until it reaches $40 \mathrm{~km} / \mathrm{h}$, and a constant pressure is then applied to the brake until the vehicle has stopped.

Fig. 3(a) presents the evolution of the vehicle's speed in each of these emergency manœuvres. The acceleration phase was

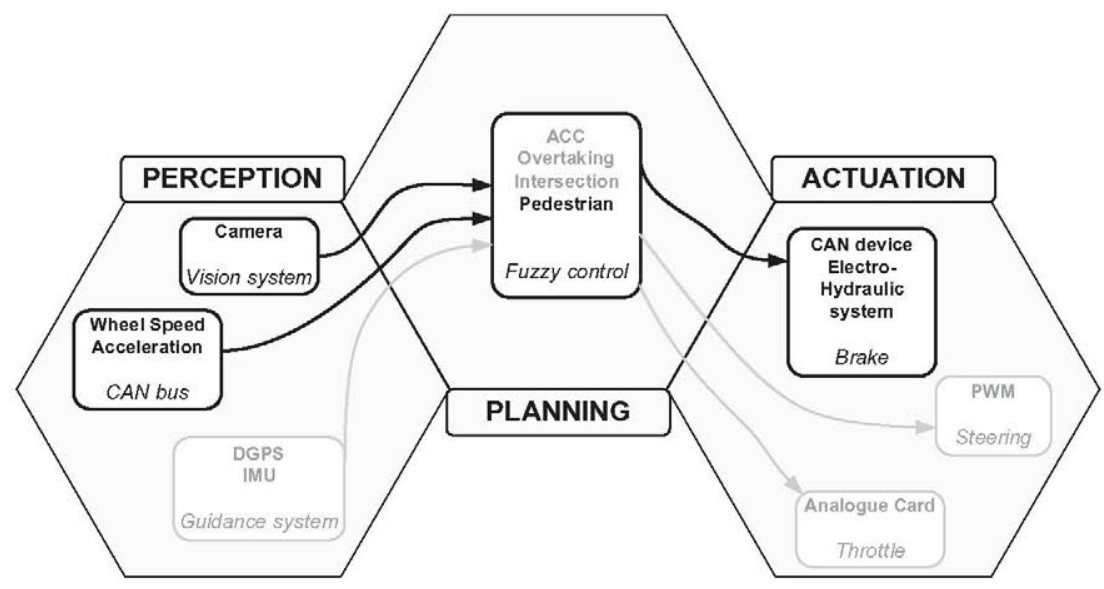

Fig. 2. Prototype vehicle control architecture. 

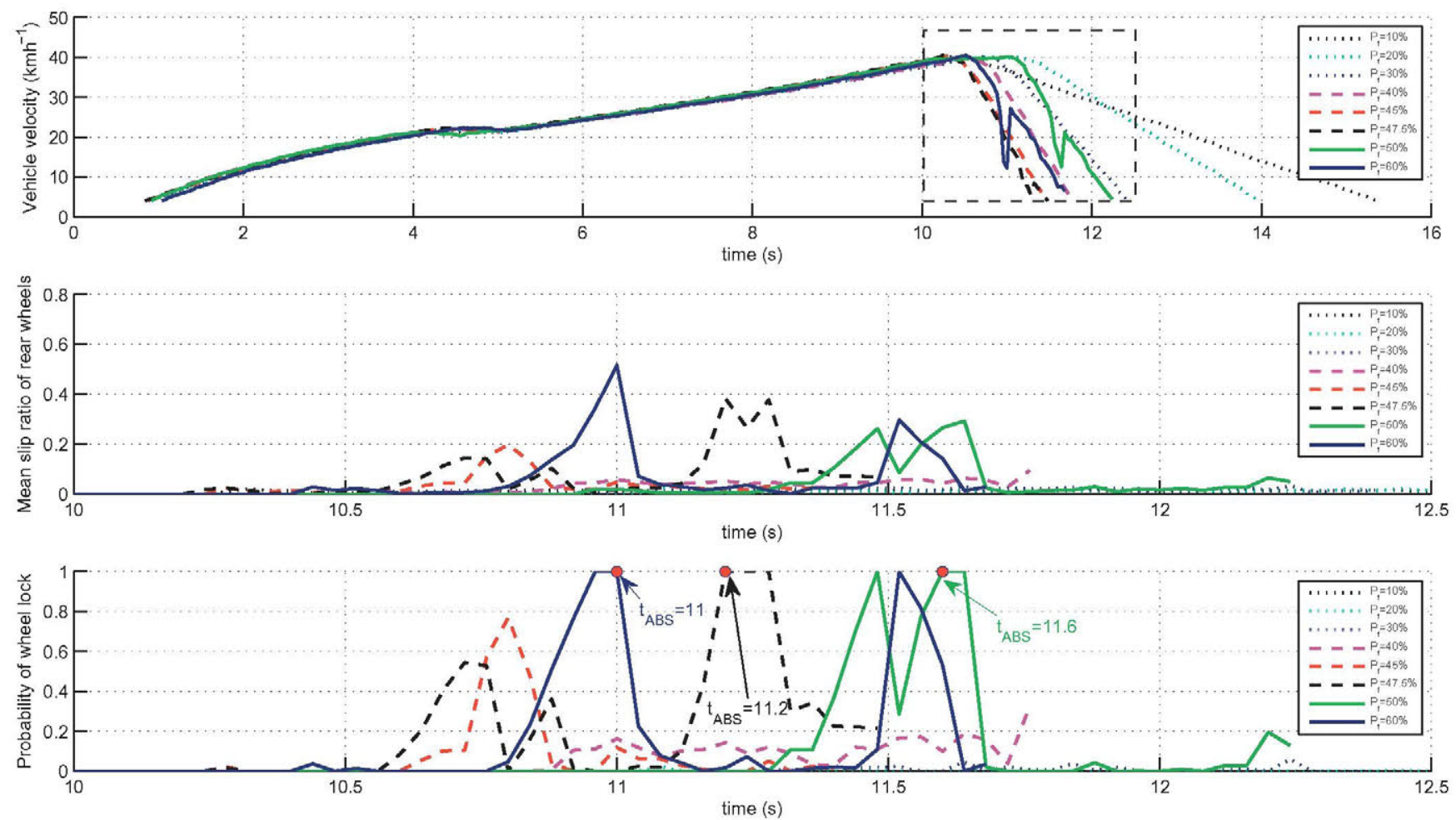

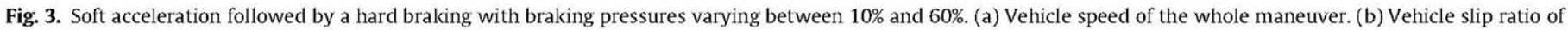
the braking phase. (c) Probability of wheel lock in the braking phase.

performed automatically in order to reduce the influence of the human driver. The greater the braking pressure applied, the steeper is the slope of the velocity. Note that speeds for $P_{f}=50 \%$ and $P_{f}=50 \%$ exhibit the classical sawtooth shape associated to the ABS activation, which shows the evolution of wheel angular velocities when the brake is rapidly hold and released. One notes also in Fig. 3(b) that the slip ratio values are more significant for the higher braking pressures. As a consequence, the slip ratios are correlated with the wheel locking probability $P_{b}$ (Fig. $3(\mathrm{c})$ ). A trade-off was made between slip ratio noise and the significant safety ranges in order to select the thresholds $\left(\tau_{\min }=0.02, \tau_{\max }=0.2\right)$ in the following function:

$$
\begin{aligned}
& P_{b}=k\left(\bar{\tau}_{\text {sat }}-\tau_{\min }\right) \\
& k=\frac{1}{\tau_{\max }-\tau_{\min }} \\
& \bar{\tau}_{\text {sat }}=\max \left(\min \left(\bar{\tau}, \tau_{\max }\right), \tau_{\min }\right)
\end{aligned}
$$

where $\bar{\tau}$ is the mean value of the right and left rear slip ratio values. Fig. 3(c) shows that the selected thresholds provide a correct match between the only three tests in which the ABS system was triggered and those in which the $P_{b}$ value is equal to unity. Furthermore, the highest probability of wheel lock is always estimated before the instant of activation of the ABS, $t_{A B S}$.

\section{Pedestrian detection system}

Pedestrian detection is carried out using the system described in Parra et al. (2007) and Llorca et al. (2009) (Fig. 4 gives an overview of the pedestrian detection architecture). Non-dense 3D maps are computed using a robust correlation process that reduces the number of matching errors (Poppe, 2007). The camera pitch angle is dynamically estimated using the so-called virtual disparity map (Llorca et al., 2009). Two main advantages result from pitch compensation. First, the accuracy of the time-to-collision estimate in car-to-pedestrian accidents is increased. Second, the separation between road points and obstacle points is improved, resulting in lower false-positive and false-negative detection rates (Llorca et al., 2009).

The 3D maps are filtered assuming the road surface to be planar (which can be acceptable in most cases), i.e., points under the actual road profile and over the actual road profile plus the maximum pedestrian height are removed since they do not correspond to obstacles (possible pedestrians). The resulting filtered 3D maps are used to obtain the ROIs.

Based on the idea that obstacles (including pedestrians) have a higher density of 3D points than the road surface, ROI selection can be carried out by determining those positions in the $3 \mathrm{D}$ space where there is a high concentration of 3D points. A 3D subtractive clustering method is proposed to handle the ROI selection stage using sparse data. The idea is to find high-density regions, which are roughly modeled by a single 3D Gaussian distribution, in the Euclidean space. The parameters of each Gaussian distribution are defined according to a minimum and maximum extent of pedestrians. Thus, while pedestrians are correctly selected, larger obstacles such as vehicles or groups of pedestrians are usually split into two or more parts. For the stereo accuracy, the method is adapted to the expected depth error (Fernández et al., 2007).

The 2D candidates are then obtained by projecting the $3 \mathrm{D}$ points of each resulting cluster, and computing their bounding boxes. A Support Vector Machine (SVM) based classifier is then applied using an optimal combination of feature-extraction methods and a by-components approach (Parra et al., 2007). While the RBF kernel provides better performance, the linear kernel is the best solution computationally. Each candidate (possible pedestrian) is divided into six regions (head, left and right arms, left and right legs, and a region between the legs). Each region is independently learned using different features. The optimal combination is obtained using texture features (Texture Unit Number) for the head and the region between the legs, histograms of gray level 


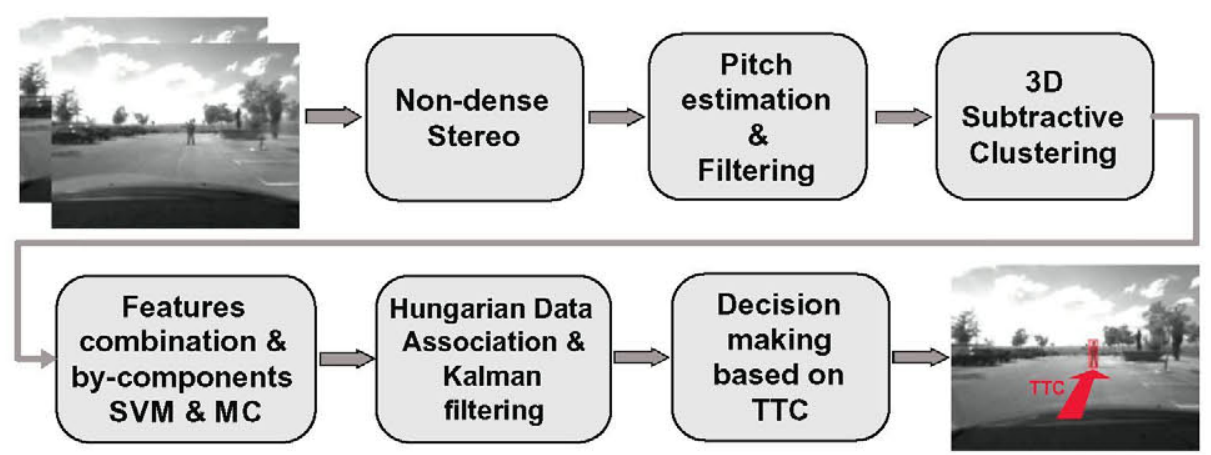

Fig. 4. Overview of the stereo vision-based pedestrian detection architecture.

differences for arms, and Histograms of Oriented Gradients (HOG) for the legs (Parra et al., 2007). The final classifier is trained with 67,650 samples $(22,550$ pedestrians and 45,100 non-pedestrians, including mirror images) obtained by a manual labeling process from sequences recorded in real traffic conditions. Our pedestrian database was firstly presented in Parra et al. (2007).

Nonetheless, the $2 \mathrm{D}$ bounding box corresponding to a $3 \mathrm{D}$ candidate might not perfectly match the actual appearance of the pedestrian in the image plane. Multiple candidates are generated around each original candidate. The so-called multi-candidate (MC) approach has been shown to increase the detection rate, the accuracy of depth measurements, and the detection range (Parra et al., 2007). The resulting pedestrians detected are tracked by means of a Kalman filter, and the data association problem is solved using the Hungarian method.

The last block of Fig. 4 represents the computation of the TTC between the host vehicle and the pedestrians detected ahead, and is defined according to the requirements of the application. For example, in the case of a driving alert application, this stage will trigger alarms to the driver depending on the host-to-pedestrian (H2P) TTC. For a pedestrian collision avoidance application, this stage will compute an input to the brake pedal controller.

\section{Control algorithm}

First the specifications of the desired behavior of the intelligent control system have to be set. Since the implementation is of an emergency system to avoid colliding with pedestrians, the primary requirement is for maximum braking pressure to be applied whenever the value of $P_{b}$ is less than $50 \%$. This requirement is reflected in the rule:

\section{R1 IF $P_{b}$ Low THEN Brake All}

The value of $P_{b}$ can change very rapidly. When it is greater than $50 \%$, the fuzzy controller modifies the pressure applied on the wheel so as to avoid locking. The variations in the fuzzy output depend on the value of the TTC, so that if the pedestrian is detected early the output pressure is less. If, however, the pedestrian is detected very close, the output pressure is increased. These changes are reflected in the following three rules:

\section{R2 IF $P_{b}$ High AND TTC Critical THEN Brake All \\ R3 IF $P_{b}$ High AND TTC Medium THEN Brake Medium \\ R4 IF $P_{b}$ High AND TTC Soft THEN Brake Nothing}

The $P_{b}$ input variable comprises two membership function definitions, one for each of its two associated linguistic labels - Low and High. The cross point between them is fixed at $75 \%$ of wheel lock probability to increase the weight of the linguistic label that represents the system reaching its physical limit when the wheels are to be blocked. The TTC has three membership function definitions according to the critical level of the detection of the pedestrian. Thus, the linguistic label Soft is used when early detection occurs, and the braking pressure is relaxed. The linguistic label Critical covers the situation when a pedestrian is detected very close to the vehicle, in particular, TTC values of less than one second are considered to constitute high risk situations, and maximum brake pressure is applied.

The output fuzzy variable is Brake. The shape of its membership function is defined using Sugeno singletons which are based on monotonic functions. Three singletons are defined with values of 1 - All, 0.5 - Medium, and 0 - Nothing, to exert the maximum, medium, or minimum pressures, respectively.

The control algorithm is represented in Fig. 5 as a control surface obtained by plotting the inferred control action Brake for a grid of values of $P_{b}$ and TTC. The appreciable smoothness in changes of slope indicate that the rules selected are appropriate.

\section{Experimental results}

\subsection{Pedestrian detection: response time and TTC estimation error}

In a first experiment, we evaluated the TTC estimation error and the response time of the pedestrian detection sensor. A set of sequences were recorded in which a driver was requested to drive along the left lane of a two-lane road at $40 \mathrm{~km} / \mathrm{h}$ and a pedestrian was requested to stand in the middle of the right lane. Besides the stereo vision sensor, two DGPSs were used, the first placed at the pedestrian's position, and the second installed onboard the vehicle. The measurements supplied by these DGPSs (after linear interpolation due to the low sampling frequency $-5 \mathrm{~Hz}$ ) were considered as the ground truth. With this experimental setup it was not necessary to carry out the emergency braking manœuvre, since both host-to-pedestrian (H2P) distance and TTC can be computed using the $\mathrm{z}$-component of the H2P vector. Fig. 6 shows a schematic view of this experiment.

The TTC estimation error is computed by dividing the depth estimation error (Rodríguez \& Aggarwal, 1990) by the vehicle speed. The speed estimation error - which is provided by the CAN bus - is assumed negligible compared to the stereo quantization error. Fig. 7 depicts the stereo and the DGPS TTC for this experiment, including the absolute error and the TTC estimation uncertainties. Some notable conclusions can be drawn from this figure. The DGPS TTC, which is considered as the ground truth, is always inside the limits of the stereo TTC plus its corresponding uncertainties. As can be observed, the larger the TTC the larger the absolute error. TTC estimates may not be reliable at long distances (absolute errors). However, the absolute error decreases as the TTC decreases. 


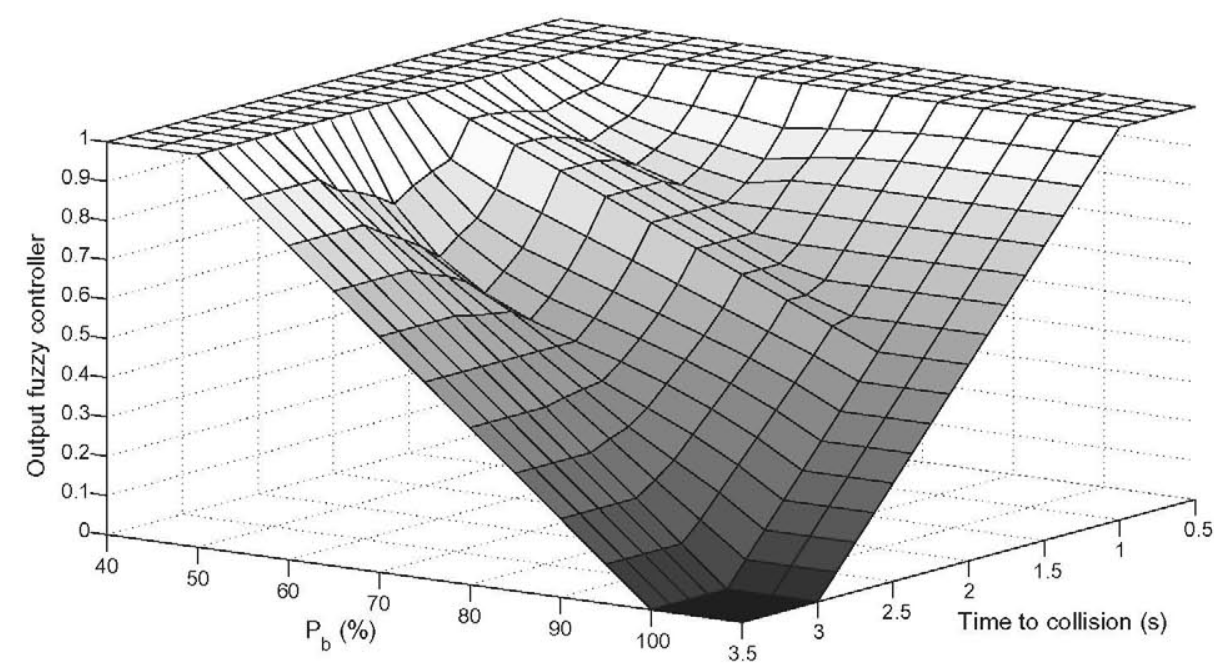

Fig. 5. Emergency pedestrian avoidance control surface.

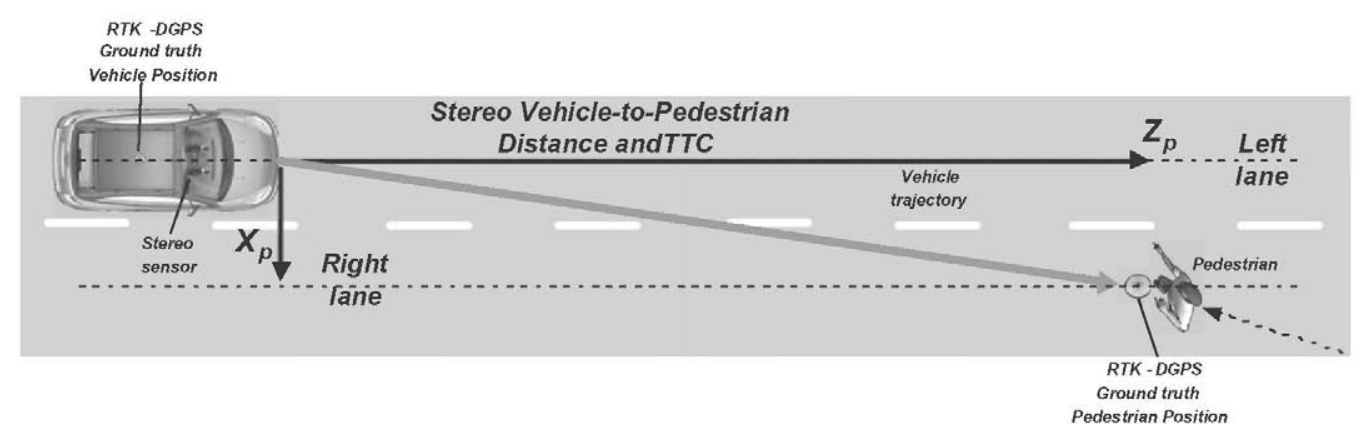

Fig. 6. Schematic view of the manual driving experimental setup.

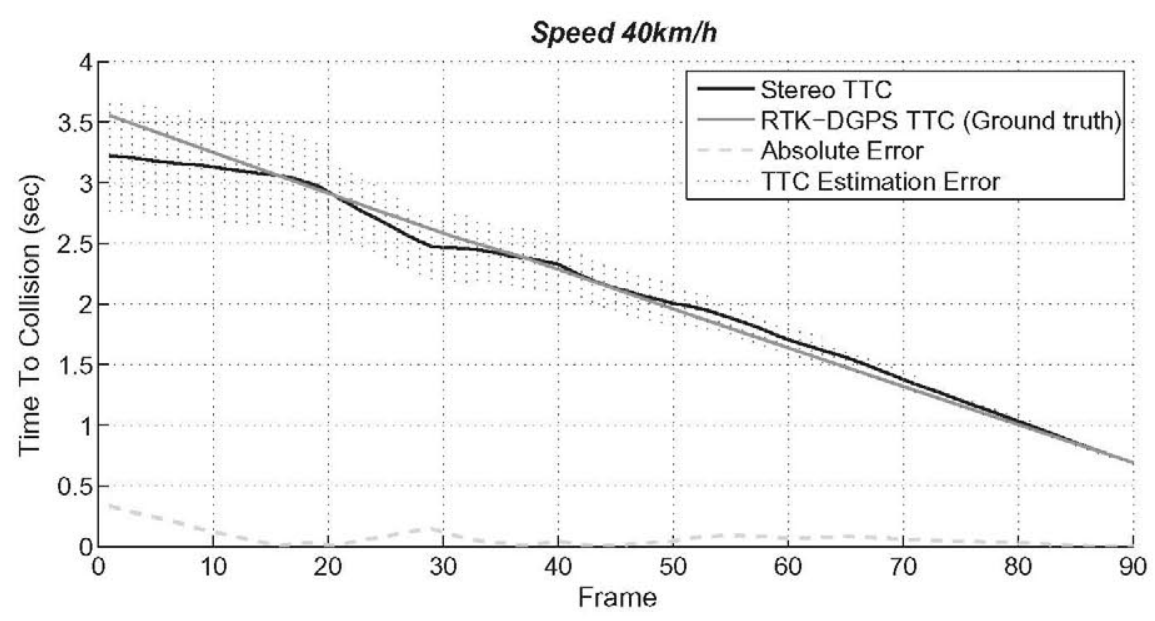

Fig. 7. Stereo and DGPS TTC, including absolute error and TTC estimation error.

The root mean square error (RMSE) of the TTC was analyzed. For TTC $<2 \mathrm{~s}$ and TTC $<1 \mathrm{~s}$, the error is reduced by factors of 1.7 and 8.5 , respectively. For the entire range of the input variable TTC of the fuzzy controller $([0,4] \mathrm{s})$, the error is less than $0.1 \mathrm{~s}$, i.e., a relative error of $2.5 \%$. This TTC estimation error is expected to have almost no influence on the controller's performance.

Human perception-reaction time (response time) for collision avoidance by braking can be defined as an interval that starts when some pedestrian enters the driver's visual field and ends when the driver has moved the foot from the accelerator to the brake pedal. It has typically been defined at around $1.5 \mathrm{~s}$ (Green, 2000; van der Horst, 1993; Olson \& Sivak, 1986; Summala, 2000). The perceptionreaction time of an automatic system can be defined as just perception time, since the reaction time can be considered negligible. The visual field of a stereo sensor is defined by the maximum range ( $35 \mathrm{~m}$ with our current sensor parameters). The bound on the 
Table 1

Number of Frames, Response Times, and Distance Needed to Detect a Pedestrian at Different Distances in the Experiments Performed at $40 \mathrm{~km} / \mathrm{h}$.

$\begin{array}{lllll}\text { Initial H2P Distance }(\mathrm{m}) & 35 & 30 & 20 & 10 \\ \text { \# of frames }(N) & 5 & 4 & 3 & 3 \\ \text { Response time }(\mathrm{s}) & 0.2 & 0.16 & 0.12 & 0.12 \\ \text { Distance }(\mathrm{m}) & 2.22 & 1.77 & 1.33 & 1.33\end{array}$

response time can be defined as RT $=N$. fps- 1 , where fps- 1 is equal to $40 \mathrm{~ms}$ (since the sensor runs at $25 \mathrm{~Hz}$ ) and $N$ is the maximum number of frames needed to detect a pedestrian. In order to determine the value of $N$, the system was run at different positions of the recorded sequence, simulating the unexpected appearance of a pedestrian at different distances. Table 1 presents the number of frames needed to detect a pedestrian, and the corresponding response time and distance traveled (note that the system needs at least three consecutive true positive classifications to start the tracking (Parra et al., 2007)). As can be observed, the greater the $\mathrm{H} 2 \mathrm{P}$ distance, the larger the value of $N$. This can be explained as being due to poorer candidate selection and classifier performance at long distances. Although the sequences were recorded under real traffic conditions, this experiment is not enough to ensure a maximum $N$ of 5 in all situations. Accordingly, we used a theoretical worst case to define a maximum value of $N=10$, which means a bound on the response time of $\mathrm{RT}=0.4 \mathrm{~s}$. This worst case value is three times faster than the typical human response time for braking. In addition, shorter response times are expected for the short distances at which the emergency stopping manœuvre is most needed.

\subsection{Autonomous intelligent emergency stopping system}

The response time and TTC estimation error are evaluated in such a way as to meet the required accuracy to perform an automatic emergency stopping manœuvre. Consequently, the visionbased pedestrian detection system together with the wheel antilock system will yield the braking pressure to apply via the fuzzy controller. For these trials, the experimental prototype vehicle described in Section 2 was used, and a lightweight dummy made of cardboard was placed in the road as the pedestrian.

Different trials were performed in order to evaluate the maximum distance required to guarantee the pedestrian's safety. A schematic view of the automatic driving experimental setup is shown in Fig. 8.

Fig. 9 shows the evolution of the relevant parameters in an emergency braking manœuvre to avoid a pedestrian collision at $40 \mathrm{~km} \mathrm{~h}^{-1}$. The top plot shows the speed of the vehicle. The second to top shows the inputs for the fuzzy controller, the gray line representing the wheel locking probability and the black one the TTC value. The next to bottom plot shows the normalized ([0-1]) fuzzy output of the applied braking pressure. The bottom plot shows the value of the distance from the vehicle to the pedestrian. The gray line represents the distance from the first frame in which a possible candidate is detected until the time when it is determined that there is indeed a pedestrian $(N=5)$. The dashed black line indicates the distance from the latter moment until the maximum value $(N=10)$ needed to avoid false emergency manœuvres. The black line shows the distance covered since the electro-hydraulic braking system started working.

One can appreciate how TTC and $P_{b}$ begin to be computed once the unexpected pedestrian has been detected. At the beginning, the braking pressure is increased quickly to unity in order to avoid the collision. When $P_{b}$ approaches unity, the braking pressure is relaxed in order to obtain the best braking distance while avoiding wheel lock. Once the value $P_{b}$ falls, the braking pressure starts to increase again to stop the vehicle in the minimum distance and in the safest way. Finally, the braking pressure is fixed at $10 \%$ to hold the vehicle stopped in case of a steep slope.

A comparison of the braking distances obtained with various constant pump pressures versus our intelligent emergency stopping system at $40 \mathrm{~km} \mathrm{~h}^{-1}$ is shown in Fig. 10. One observes that the control system avoids wheel lock, and provides a satisfactory stopping distance when compared to constant pressure braking in an ABS-equipped vehicle. Note that, even if the braking distance is not less than that obtained with $P_{f}=60 \%$, our control system still exhibits remarkable behavior for such a low sample rate compared with that used in commercial ABS systems (which usually work at between 100 and $250 \mathrm{~Hz}$ ). Consequently, the proposed intelligent braking system could be used in any mass production car (with or without $\mathrm{ABS}$ ).

Finally, Table 2 summarizes the mean results obtained at $40 \mathrm{~km} \mathrm{~h}^{-1}$ and $50 \mathrm{~km} \mathrm{~h}^{-1}$ with different estimated $\mathrm{H} 2 \mathrm{P}$ distances, giving the real braking distances, the minimum detection distances - braking distance plus distance covered with $N=5$, and the safe detection distances - braking distance plus distance covered with $N=10$.

\section{Conclusions and future work}

This paper has described an autonomous driving system capable of working in parallel with a human driver in order to carry out an automatic pedestrian avoidance manœuvre involving braking action. It consists of: vision-based pedestrian detection to calculate the vehicle-pedestrian distance which, combined with the vehicle's speed obtained via a CAN bus, allows the TTC to be determined; estimation of the wheel locking probability; and a fuzzy controller that uses these two values as inputs to generate the braking pressure to be applied in order to avoid collision with the pedestrian.

The system was mounted on a Citroën C3 Pluriel car in which an autonomous electro-hydraulic braking system had been installed to be used in parallel with the original circuit. The vision-based system is located close to the rear-view mirror, and the rest of the input variables are obtained via the CAN bus of the vehicle.

Several trials conducted at urban speeds - up to $50 \mathrm{~km} \mathrm{~h}^{-1}$ - on a real circuit showed the good behavior of the system. We believe that the designed system will represent an encouraging starting point for the development of automatic devices that can act on

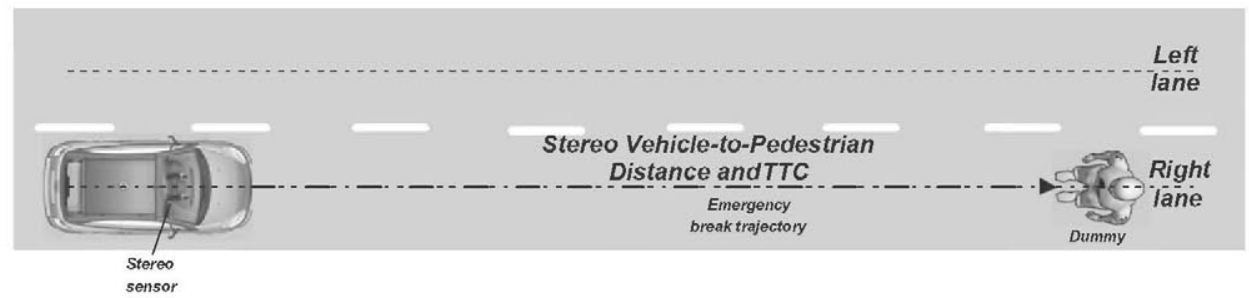

Fig. 8. Schematic view of the automatic driving experimental setup. 

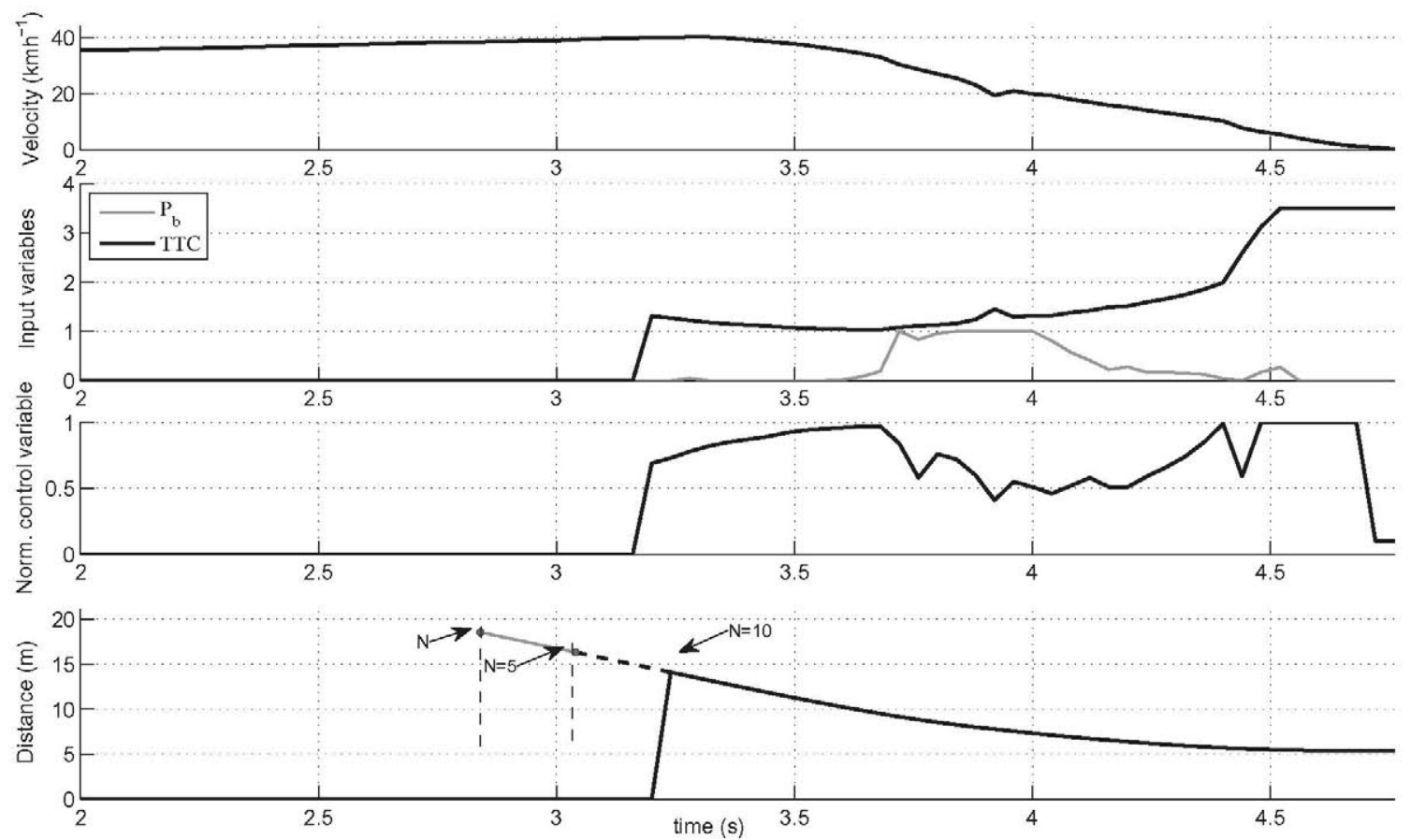

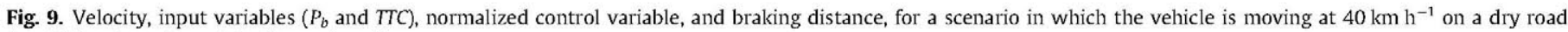
$(\mu=0.9)$ and an obstacle is detected at $13 \mathrm{~m}$.
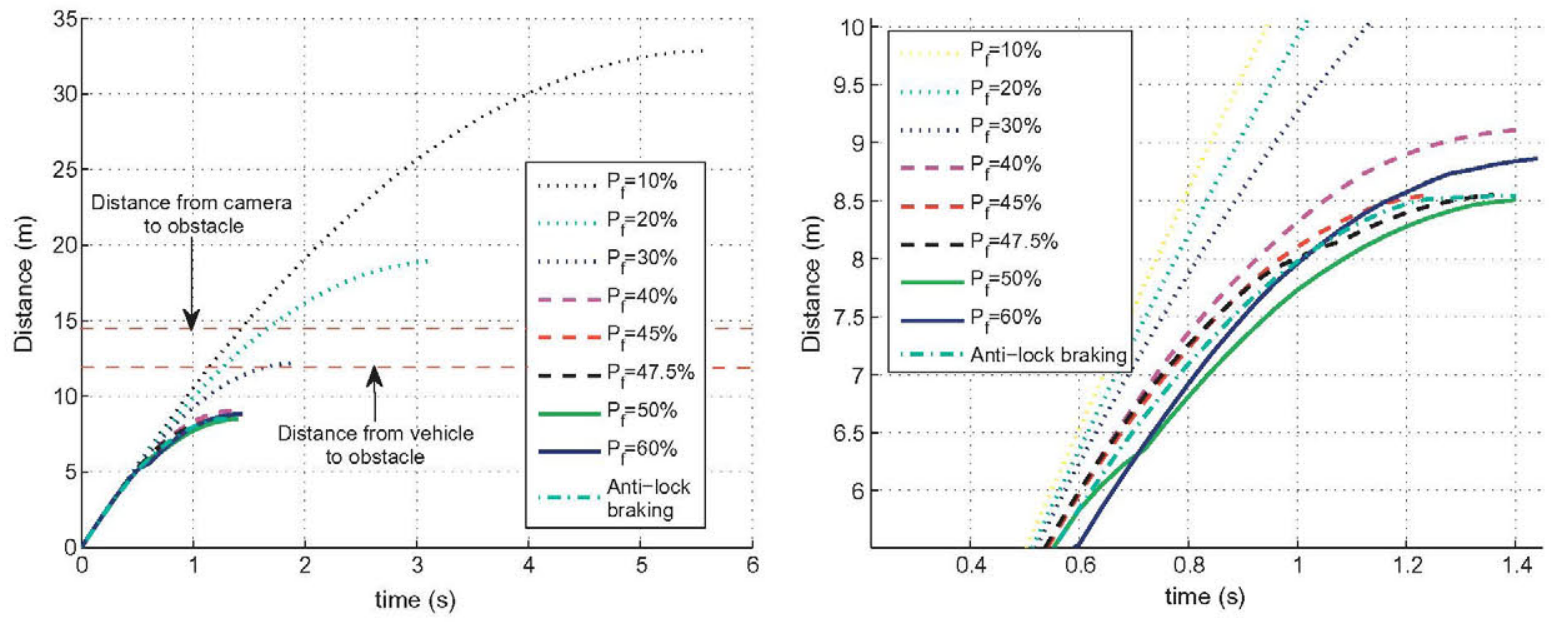

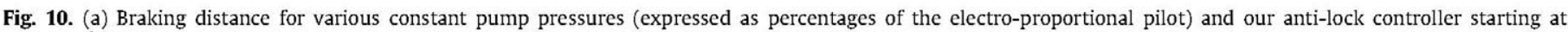
$40 \mathrm{~km} \mathrm{~h}^{-1}$. (b) Detail of part of the top diagram.

Table 2

Mean braking distance results.

\begin{tabular}{lllll}
\hline Speed $\left(\mathrm{km}^{-1}\right)$ & 40 & & 50 & \\
\hline H2P distance $(\mathrm{m})$ & 22 & 17 & 25 & 22 \\
Safe detection distance $(\mathrm{m})$ & 13.91 & 13 & 20.56 & 19.30 \\
Minimum detection distance $(\mathrm{m})$ & 11.69 & 10.78 & 17.78 & 16.52 \\
Braking action distance $(\mathrm{m})$ & 9.47 & 8.56 & 15 & 13.74 \\
\hline
\end{tabular}

the car independently of the driver when a possible collision with a pedestrian is detected by some means, and all the warnings have failed to produce the driver's response.

As future work, the behavior of the system under different weather conditions (such as fog for the vision system, or snow for the wheel locking probability) will be studied so as to include a new fuzzy variable that will take the effects of these environmental conditions into account in our controller.

\section{Acknowledgment}

This work was financed by the Spanish Ministry of Economy and Competitiveness under Research Grant ONDA-F TRA201127712.

\section{References}

Akbarzadeh, M., Emami, K., \& Pariz, N. (2004). Adaptive discrete-time fuzzy sliding mode control for anti-lock braking systems. In Proceedings of the annual meeting NAFIPS, New Orleans, LA (pp. 554-559).

Castro, J., Delgado, M., \& Medina, M. R.-L. J. (2011). An expert fuzzy system for predicting object collisions. Its application for avoiding pedestrian accidents. Expert Systems with Applications, 38, 486-494. 
Chang, B., Tsai, T., \& Young, C. (2010). Intelligent data fusion system for predicting vehicle collision warning using vision/GPS sensing. Expert Systems with Applications, 37, 2439-2450.

Commission, E. T. (2009). Final review of the European Commission's 2001 Transport white paper. A sustainable future for transport. Technical report. European Commission.

Enzweiler, M., \& Gavrila, D. (2003). Monocular pedestrian detection: Survey and experiments. IEEE Transactions on Pattern Analysis and Machine Intelligence, 31, 2179-2195.

Fernández, D., Parra, I., Sotelo, M. A., Revenga, P., Álvarez, S., \& Gavilăn, M. (2007). 3D candidate selection method for pedestrian detection on non-planar roads. In: Proceedings of the IEEE intelligent vehicle symposium.

Gandhi, T., \& Trivedi, M. M. (2007). Pedestrian protection systems: Issues, survey, and challenges. IEEE Transactions on Intelligent Transportation Systems, 8 413-430.

Gerónimo, D., López, A., Sappa, A., \& Graf, T. (2010). Survey on pedestrian detection for advanced driver assistance systems. IEEE Transactions on Pattem Analysis and Machine Intelligence, 32, 1239-1258.

Gidel, S., Checchin, P., Blanc, C., Chateau, T., \& Trassoudaine, L. (2010). Pedestrian detection and tracking in an urban environment using a multilayer laser scanner. IEEE Transactions on Intelligent Transportation Systems, 11, 579-588.

Green, M. (2000). How long does it take to stop? Methodological analysis of driver perception-brake times. Transportation Human Factors, 2, 195-216.

Jamshidi, M., Vadiee, N., \& Ross, T. (1993). Fuzzy logic and control: Software and hardware applications. Prentice-Hall.

Kayacan, E., Oniz, Y., \& Kaynak, O. (2009). A grey system modeling approach for sliding-mode control of antilock braking system. IEEE Transactions on Industrial Electronics, 56, 3244-3252.

Lee, Y., \& Zak, S. (2001). Genetic neural fuzzy control of anti-lock brake systems. In Proceedings of the American control conference, Arlington, VA (pp. 671-676).

Liu, C.-s. (2008). Adhesion coefficient of automobile tire and road surface. Journal of Central South University of Technology, 15, 210-214. http://dx.doi.org/10.1007/ s11771-008-0348-5.

Llorca, D. F., Sotelo, M. A., Parra, I., Naranjo, J. E., Gavilắn, M., \& Álvarez, S. (2009). An experimental study on pitch compensation in pedestrian-protection systems for collision avoidance and mitigation. IEEE Transactions on Intelligent Transportation Systems, 10, 469-478.

Mauer, G. (1995). A fuzzy logic controller for an ABS braking system. IEEE Transactions on Fuzzy Systems, 3, 381-388.

Milanés, V., Pérez, J., Godoy, J., \& Onieva, E. (2012). A fuzzy aid rear-end collision warning/avoidance system. Expert Systems with Applications, 39, 9097-9107.

Milanés, V., González, C., Naranjo, J., Onieva, E., \& de Pedro, T. (2010). Electrohydraulic braking system for autonomous vehicles. International Joumal of Automotive Technology, 11, 1-6.
Milanés, V., Llorca, D., Villagrá, J., Pérez, J., Fernández, C., Parra, l., et al. (2012) Intelligent automatic overtaking system using vision for vehicle detection. Expert Systems with Applications, 39, 3362-3373.

Milanés, V., Naranjo, J., González, C., Alonso, J., \& Pedro, T. (2008). Autonomous vehicle based in cooperative GPS and inertial systems. Robotica, 26, 627-633.

Milanés, V., Pérez, J., Onieva, E., \& González, C. (2010). Controller for urban intersections based wireless communications and fuzzy logic. IEEE Transactions on Intelligent Transportation Systems, 11, 243-248.

Moeslund, T. B., Hillton, A., \& Krüger, V. (2006). A survey of advances in vision-based human motion capture and analysis. Computer Vision and Image and Understanding, 104, 90-126.

Murphy, R. R. (2000). Introduction to Al robotics. Cambridge, MA: MIT Press.

N.C. for Statistics, Analysis (2006). Motor vehicle traffic crash fatality counts and estimates of people injured for 2005. Technical Report. US: Department of Transportation.

Olson, P. L., \& Sivak, M. (1986). Perception-response time to unexpected roadway hazards. Human Factors, 28, 91-96.

Onieva, E., Milanés, V., González, C., de Pedro, T., Pérez, J., \& Alonso, J. (2010) Throttle and brake pedals automation for populated areas. Robotica, 28, 509-516.

Parra, I., Llorca, D. F., Sotelo, M. A., Bergasa, L. M., Revenga, P., Nuevo, J., et al. (2007) Combination of feature extraction methods for SVM pedestrian detection. Transactions on Intelligent Transportation Systems, 8, 292-307.

Poppe, R. (2007). A vision-based human motion analysis: An overview. Computer Vision and Image and Understanding, 108, 4-18.

Rodríguez, J. J.. \& Aggarwal, J. K. (1990). Stochastic analysis of stereo quantization error. IEEE Transactions on Pattern Analysis and Machine Intelligence, 12, 467-470.

Su, S., Chang, J., \& Chen, S. (2006). The study on direct adaptive fuzzy controllers International Journal of Fuzzy Systems, 8, 150-159.

Summala, H. (2000). Brake reaction times and driver behavior analysis. Human Factors, 2.

van der Horst, R., \& Hogema, J. (1993). Time-to-collision and collision avoidance systems. In Proceedings of the 6th ICTCT workshop: Safety evaluation of traffic systems: Traffic conflicts and other measures (pp. 109-121).

Wang, W., Chen, G., \& Tao, C. (2003). Stable anti-lock braking system using outputfeedback direct adaptive fuzzy neural control. In Proceedings of the IEEE international conference on systems, man, and cybernetics (pp. 3675-3680).

Wang, W., Li, I., Chen, M., Su, S., \& Hsu, S. (2009). Dynamic slip-ratio estimation and control of antilock braking systems using an observer-based direct adaptive fuzzy-neural controller. IEEE Transactions on Industrial Electronics, 56, $1746-1756$.

Zanten, A., Erhardt, R., \& Lutz, A. (1990). Measurement and simulation of transients in longitudinal and lateral tire forces. SAE Transactions, 99, 300-318. 